de la recherche clinique, en particulier, il est urgent d'évaluer la pertinence et la qualité des gestes délivrés. Il est urgent de proposer ce défi aux nutritionistes, physiothérapeutes et techniciens de toutes sortes (y compris les administrateurs du système de santé) impliqués dans la distribution de soins. Au Québec, le Fonds de la recherche en santé (FRSQ) s'est donné pour tâche d'y parvenir en dix ans. Défi des années 1990 ! Nous comptons sur une collaboration et une concertation entre l'État et l'Université, dans le cadre du développement d'hôpitaux universitaires dont la mission englobe de facto les trois volets de soins, d'enseignement et de recherche et ce, pour chaque catégorie de professionnels de la santé qui y œuvrent.

Il est aussi urgent de recourir largement aux développements techniques survenant dans des domaines apparemment loin de la santé, pour en extraire les retombées potentiellement positives. Nous comptons sur une collaboration et une concertation entre l'Université et l'Industrie des biens de santé (pharmaceutiques, instruments médicaux). En effet, le progrès dans ce domaine dépend de la mise au point d'évolutions techniques et scientifiques (vocation universitaire) comme de la démonstration de l'impact bénéfique de ces nouveaux produits sur la santé de l'homme (vocation hospitalière). Nous comptons enfin sur les appareils étatiques qui gèrent la délivrance des biens de santé. Ils doivent se préoccuper de l'évaluation systématique de leurs opérations dans un contexte d'objectivité : c'est-à-dire en ayant recours à des équipes de chercheurs indépendants. Ils doivent contribuer à créer cette infrastructure d'experts compétents. Ils doivent se tourner vers de nouvelles préoccupations de prévention, souvent oubliées au profit de l'escalade des coûts, et faire place aux nouvelles perspectives apportées par la génétique. Enfin, nous comptons sur la diffusion rapide des progrès de la connaissance et sur la vulgarisation respectueuse des perspectives qu'ils nous ouvrent. La mission de revues comme médecine/sciences est aussi d'exposer à tous cette révolution permanente de la santé aux multiples facettes

$m / s n^{\circ} 3$ vol. 6 , mars 90

\section{Presse scientifique et industrie pharmaceutique}

L'industrie du médicament est une industrie fondée sur la recherche. Le monde économique en est persuadé, et il n'est que de suivre l'évolution de ce secteur d'activité pour constater que les résultats de la recherche constituent sa véritable matière première. L'une des composantes essentielles de cette recherche est l'avancée, maintenant spectaculaire, dans la connaissance des mécanismes intimes cellulaires et subcellulaires. A la période de recherche par screening des activités potentielles de telle ou telle molécule succède une période où la rationalité de la recherche est plus importante du fait des relations de causalité des désordres à l'origine ou cause des maladies, maintenant étudiées. Il en résulte que l'industrie a besoin d'une information permanente, de haut niveau scientifique et fiable quant à sa consistance, non seulement sur sa propre recherche, mais sur celle qui est faite en dehors de la firme. C'est de cette information que résultent désormais l'orientation des programmes, la hiérarchisation des sujets et finalement l'attribution des moyens qui engagent l'avenir des firmes. On peut dire sans risque de se tromper qu'aujourd'hui la connaissance des résultats scientifiques, où qu'ils aient pris naissance dans le monde, constitue un élément de la politique industrielle dont aucun dirigeant ne peut se passer pour ce qui concerne sa firme.

En outre, la recherche du médicament s'apprécie de plus en plus en termes de risques en raison de son coût élevé et de la durée nécessaire à la mise sur le marché d'un médicament. Une mauvaise orientation, parce que dépassée scientifiquement, doit pouvoir être corrigée très tôt sous peine de conséquences qui peuvent être dramatiques. La fonction "évaluation" est ainsi devenue une part intrinsèque de la recherche et, pour s'exercer avec fruit, ne peut résulter que de la connaissance par des publications et revues de l'état des connaissances partout dans le monde.

Cette place nouvelle des publications et ouvrages scientifiques au service de l'industrie ne peut être atteinte que si un certain nombre de conditions sont convenablement remplies.

- Qualité des publications : la notoriété atteinte par certaines publications n'est pas suffisante à elle seule. Il convient sans aucun doute de favoriser l'émergence, pour maintenir la compétition et éviter tout malthusianisme, de revues nouvelles ou de favoriser l'évolution de revues existantes, en admettant sans doute une certaine spécialisation. - Nature des publications : de nouvelles préoccupations prennent une place prépondérante dans le besoin d'informations de qualité des industriels. C'est le cas des problèmes d'économie de santé. Au moment où l'opinion récuse de plus en plus, et à juste titre, un rationnement des soins inhérent aux problèmes de financement de la protection sociale, il est nécessaire que des recherches soient conduites, publiees et confrontées dans un domaine où la hiérarchisation des besoins et donc le choix des moyens deviennent inéluctables. La recherche, nécessairement inclusive de l'éthique médicale, est, comme dans d'autres domaines, inséparable du progrès d'ensemble à assurer et des méthodologies à mettre en auvre.

Les industriels du médicament seront nécessairement attentifs à voir ces besoins satisfaits. Ils souhaitent que le caractère de haute tenue scientifique que doit avoir une telle presse résulte d'une compétition fondée sur la qualité, d'où sortiront la notoriété et l'équilibre économique de cette presse. Ils sont conscients qu'il s'agit d'une auvre de longue haleine.

René Sautier

Président du Syndicat national de l'industrie pharmaceutique 